Electronic Supporting Information

\title{
Wearable Collector for Noninvasive Sampling of SARS-CoV-2 from Exhaled Breath for Rapid Detection
}

Fernando Soto ${ }^{1,2}$, Mehmet Ozgun Ozen ${ }^{1,2}$, Carlos F. Guimarães 1,2,3,4, Jie Wang',2, Kallai Hokanson ${ }^{1,2}$, Rajib Ahmed ${ }^{1,2}$, Rui L. Reis ${ }^{3,4}$, Ramasamy Paulmurugan ${ }^{2,5}$, Utkan Demirci $^{1,2^{*}}$

${ }^{1}$ Bio-Acoustic MEMS in Medicine (BAMM) Laboratory, Canary Center at Stanford for Cancer Early Detection, Department of Radiology, School of Medicine Stanford University, Palo Alto, California.

94304-5427, USA.

${ }^{2}$ Canary Center at Stanford for Cancer Early Detection, Department of Radiology, School of Medicine, Stanford University, Palo Alto, California 94304-5427, USA

33B's Research Group - Research Institute on Biomaterials, Biodegradables and Biomimetics, University of Minho, Headquarters of the European Institute of Excellence on Tissue Engineering and Regenerative Medicine, 4805-017 Guimarães, Portugal.

${ }^{4}$ ICVS/3B's - Portuguese Government Associate Laboratory, University of Minho, Braga and Guimarães, Portugal. ${ }^{5}$ Molecular Imaging Program at Stanford (MIPS), Department of Radiology, School of Medicine, Stanford University, Palo Alto, California 94304-5427, USA.

*Corresponding author. Email: utkan@stanford.edu

\section{Table of contents}

\section{Supporting Video Description}

Video S1. Examination of its resiliency to external mechanical strain of a wearable collector attached to a surgical mask.

Video S2. Fluorescent aerosolized droplets generation using a spray atomizer directed at the collector attached to a surgical mask. 


\section{Modelled collection of viruses}

Simulation: The capture of the virus by the collector was modeled using MATLAB. The model was based on the 3D movement of respiratory particles carrying the virus through the air. Euler's method was used to determine the location of particles and impact behavior (rebounding, splashing, or adhesion) was determined by the individual droplet's Laplace number (Equation 1) and Weber number (Equation 2)

$$
\begin{gathered}
W e=\frac{\rho_{f} U_{f}^{2} d_{p}}{\sigma_{f}} \\
L a=\frac{\rho_{f} U_{f}^{2} d_{p}}{\mu_{f}^{2}}
\end{gathered}
$$

The mask's resistance to flow was accounted for by the pressure difference across the material where that pressure difference is defined by equation 3 . The difference across the material must equal the pressure difference across the gap in the mask which is the combined pressure loss at the gap inlet, outlet and within the gap itself (equation 4, average velocity of air through the mask : $v_{m}$ density of air: $\rho=1.225 \mathrm{~kg} / \mathrm{m}^{3}$, viscous porous resistance $C_{m}=2000 \mathrm{~m} / \mathrm{s}^{2}$, loss coefficient $\zeta=\zeta_{\text {in }}+$ $\zeta_{\text {out }}=1.5$, average velocity of air escaping the mask through the gap: $v_{g}$, Gap distance at the edge of mask: $H_{g}$, gap width: $B_{g}$, length of the gap: $L_{g}$ ).

$$
\begin{gathered}
\Delta p=p_{m}-p_{a} \approx C_{m} \rho v_{m} \\
C_{m} \rho v_{m}=\frac{v_{t}}{\left|v_{t}\right|} \frac{\zeta \rho}{2} v_{g}^{2}+\frac{12 \mu L_{g}}{H_{g}^{2}} v_{g}
\end{gathered}
$$

The boundary values for the particles were determined by the flow through the mask (equation 6 , mask surface area: $A_{m}$ ) and the gap between the face and mask (equation 7) where the total flow $F_{t}$ 
(equation 5). The velocity of the air through the mask and the velocity through the mask gap (equation 3).

$$
\begin{aligned}
& F t=F g+F m \\
& F m=A m * v m \\
& F g=v g H g B g
\end{aligned}
$$

Breathing was represented by a cyclical time-varying average initial velocity of respiratory particles. The collector was modeled as a porous circular disk (diameter $=2.5 \mathrm{~cm}$, thickness $=10$ $\mu \mathrm{m}$, pore density $=3 * 10^{12}$ pores $/ \mathrm{m}^{2}$, pore diameter $=200 \mathrm{~nm}$ ) placed directly in front of the mouth (gap between mouth and collector $=.5 \mathrm{~cm}$ ) which was assumed to be the only breath source. The percentage of viruses that land on the collector was determined by dividing the total number of viruses within the pores and the surface of the collector divided by the total number of viruses expelled in the model. 


\section{Supporting Figures}

a
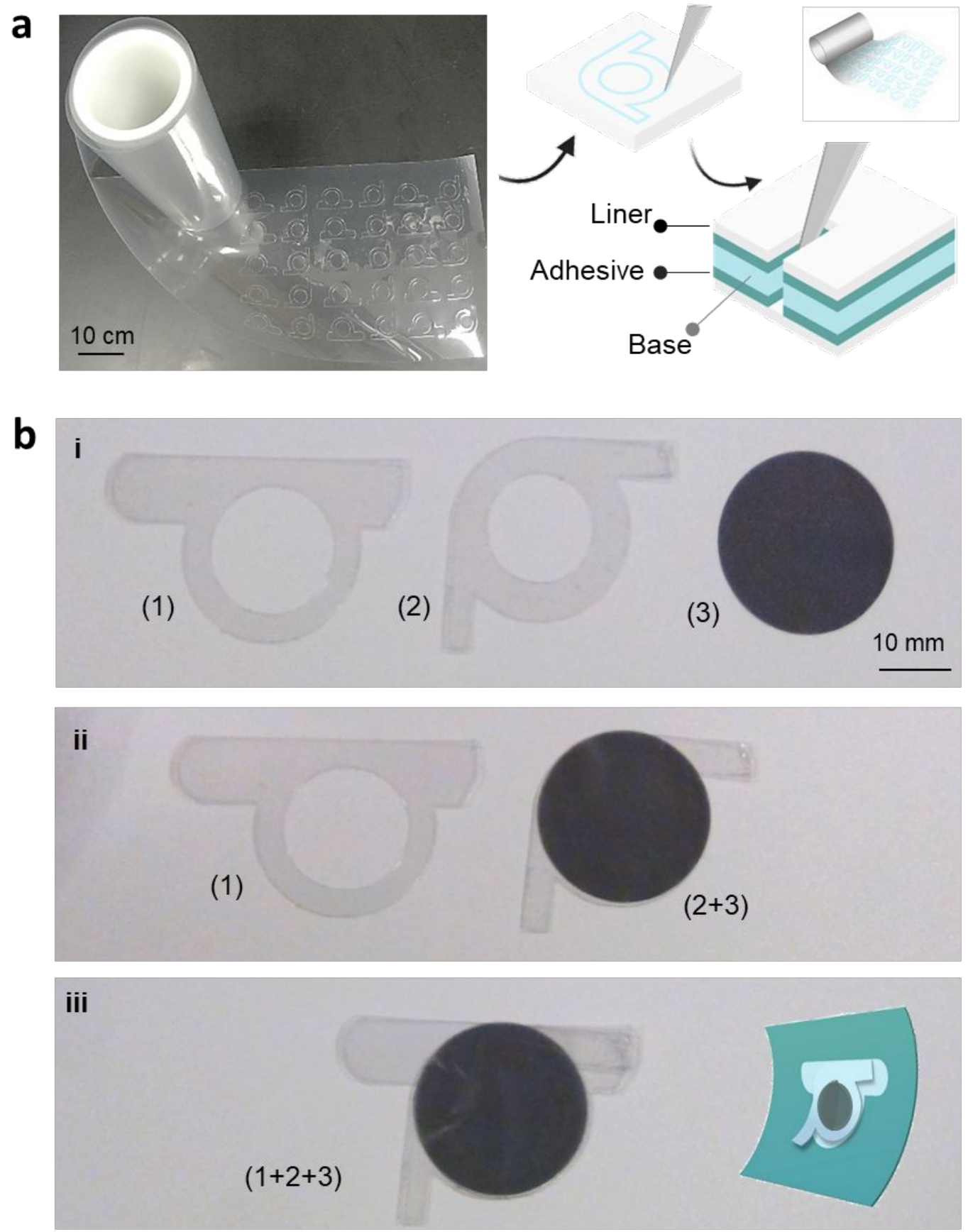

Figure S1. (a) Fabrication of the adhesive is performed via die cutting a roll of five layers consisting of liner-adhesive-base-adhesive-liner. (b) Assembly steps (i-iii) of the three components required to build the wearable patch including (1) adhesive $A$, (2) adhesive $B$, and (3) porous membrane. 
a

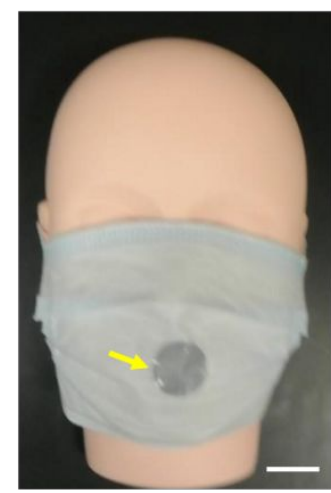

b

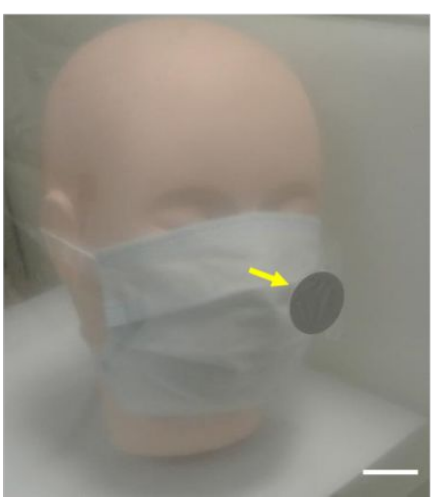

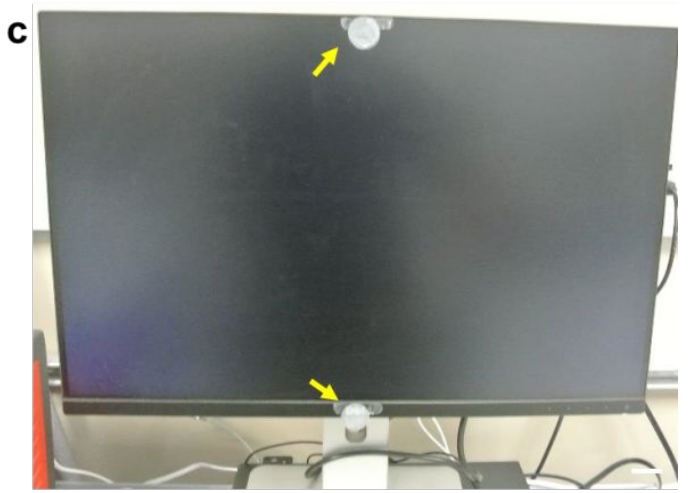

Figure S2. Adhesive collector for sampling a) outside the mask, b) face cover, and c) computer monitor. Scale bars, $25 \mathrm{~mm}$.

a

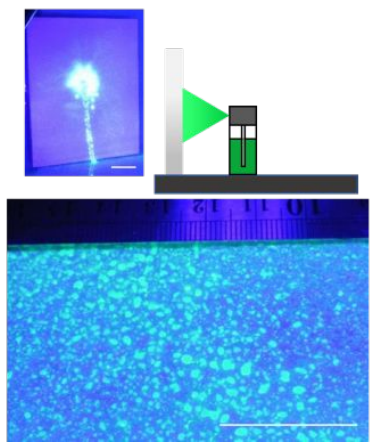

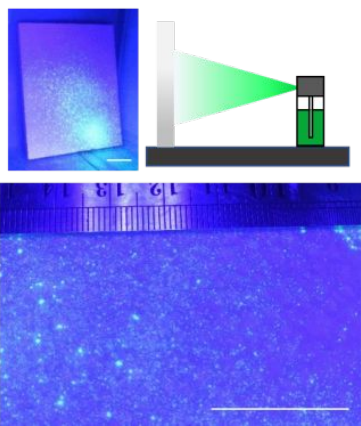

b
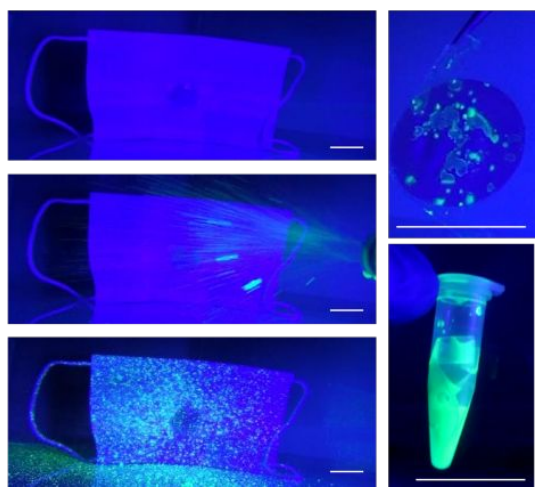

Figure S3. a) Fluorescent aerosolized of droplets generation based on different distance of sprayer to objective. b) Fluorescent visual model to identify landing of spray (before, during and after spraying), inset illustrates patch collector cover with fluorescent dye and transfer to a collection tube. Scales, bar $25 \mathrm{~mm}$.

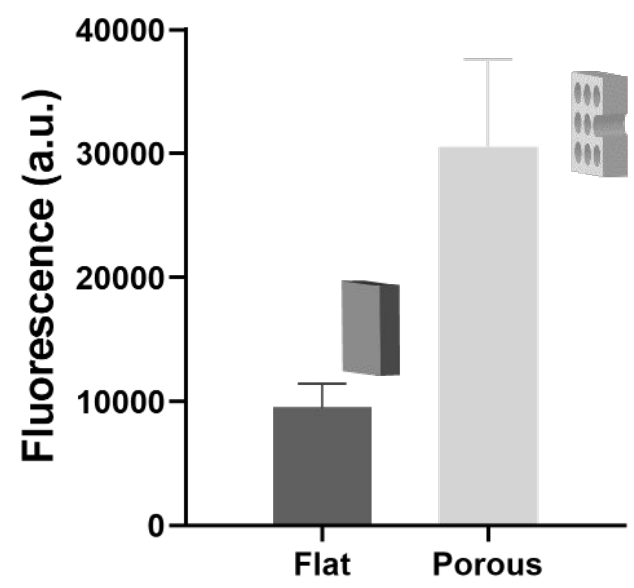

Figure S4. Fluorescence intensity comparing dye collected in a flat vs $200 \mathrm{~nm}$ porous surface after spraying dye with atomizer. 
a
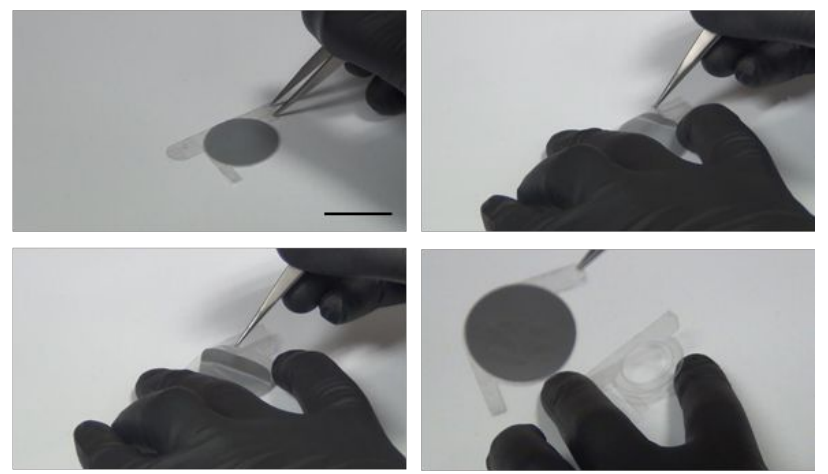

b
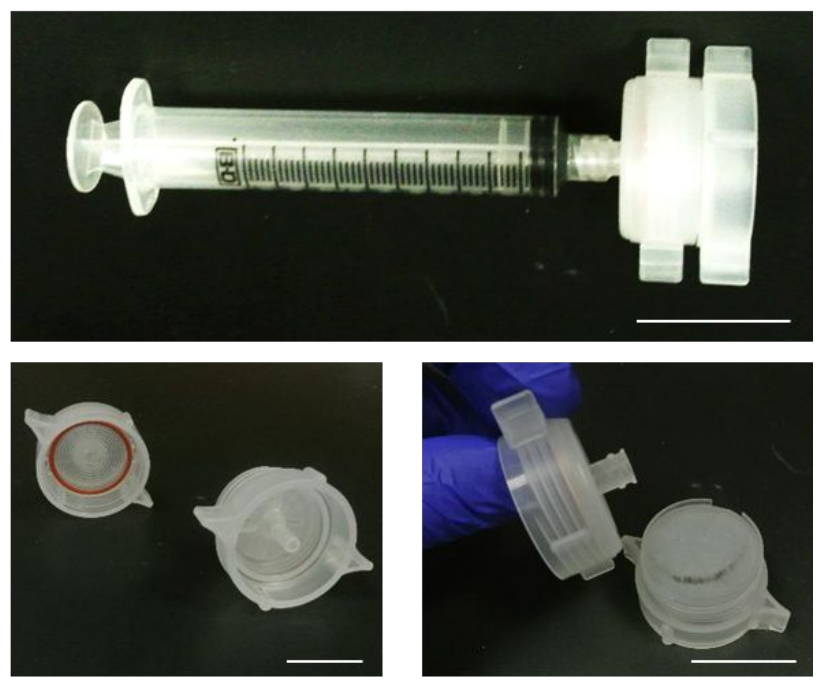

Figure S5. a) Disassembly of wearable collector and b) use of reusable syringe filter to recover collected viruses. Scale bars, $25 \mathrm{~mm}$. 